\title{
Cardiac Abnormalities in COVID-19 Patients: Should a Cardiac Echocardiogram be Routine?
}

\author{
Carina Ramalho, Mariana Almeida, Francisco Gomes, Magda Silva, Silvia Rodrigues \\ Centro Hospitalar Barreiro Montijo, Barreiro, Portugal
}

Doi: 10.12890/2021_002559- European Journal of Case Reports in Internal Medicine - 으 EFIM 2021

Received: 13/04/2021

Accepted: 28/04/2021

Published: 14/05/2021

How to cite this article: Ramalho C, Almeida M, Gomes F, Silva M, Rodrigues S. Cardiac abnormalities in COVID-19 patients: should a cardiac echocardiogram be routine? EJCRIM 2021;8: doi:10.12890/2021_002559.

Conflicts of Interests: The authors declare there are no competing interests.

This article is licensed under a Commons Attribution Non-Commercial 4.0 License

\section{ABSTRACT}

Current scientific evidence shows that SARS-CoV-2 infection is associated with an increased risk of thromboembolic events. In patients with ischaemic heart disease and heart failure, thrombi of the left ventricle can increase patient mortality, mainly due to the risk of systemic embolization. Given the hypercoagulable state associated with COVID-19, such events may be more likely. We describe a patient hospitalized for congestive heart failure and SARS-CoV-2 infection who was diagnosed with a thrombus in the left ventricle. After the thrombus was identified on echocardiography and treated with anticoagulation, it completely resolved and cardiac function improved.

\section{LEARNING POINTS}

- SARS-CoV-2 infection has been frequently associated with thromboembolic phenomena and intracardiac thrombi.

- Echocardiographic evaluation in patients with SARS-CoV-2 infection is important, especially in cases with known or new-onset cardiac pathology which predisposes to thromboembolic phenomena.

- The timely diagnosis and treatment of intracardiac thrombi can prevent complications and improve patient prognosis.

\section{KEYWORDS}

SARS-CoV-2, COVID-19, left ventricular thrombus, heart failure

\section{CASE DESCRIPTION}

We present the case of a 50-year-old man with a history of type 2 diabetes and dyslipidaemia. He attended the emergency department with complains of cough and dyspnoea with 1 week of evolution. He was feverish, with an overall decrease in breath sounds and oedema of the lower limbs. Blood analysis showed an increase in inflammatory parameters (CRP $64.1 \mathrm{mg} / \mathrm{dl}$ ), acute kidney failure (urea $64 \mathrm{mg} /$ $\mathrm{dl}$, creatinine $1.65 \mathrm{mg} / \mathrm{dl}$ ), a NT-proBNP of $30.391 \mathrm{pg} / \mathrm{ml}$ and an increase in high-sensitivity troponins (1st dose: $628.1 \mathrm{pg} / \mathrm{ml}, 2 \mathrm{nd}$ dose: $1345 \mathrm{pg} / \mathrm{ml}$ ). The EKG showed left axis deviation, with no signs of acute ischaemia. Chest x-ray showed opacity in the right pulmonary field, while a CT scan of the chest showed increased cardiac dimensions, discreet pericardial effusion, mild to moderate bilateral pleural effusion and signs of ascites. The patient was admitted with new-onset dilated cardiomyopathy of unknown aetiology with poor systolic function. Neurohormonal therapy was started. The patient underwent an RT-PCR SARS-CoV-2 test which was positive, and hydroxychloroquine, ceftriaxone and azithromycin were started, given the elevated inflammatory parameters and suspicion of bacterial infection.

A transthoracic echocardiogram was performed on the third day of hospitalization and showed severe left ventricle dilation and dysfunction with a $15 \%$ ejection fraction (eyeball estimation) and, at the level of the septum/apex, a $33 \times 12 \mathrm{~mm}$ mass with a mobile and friable appearance (Fig. 1). 


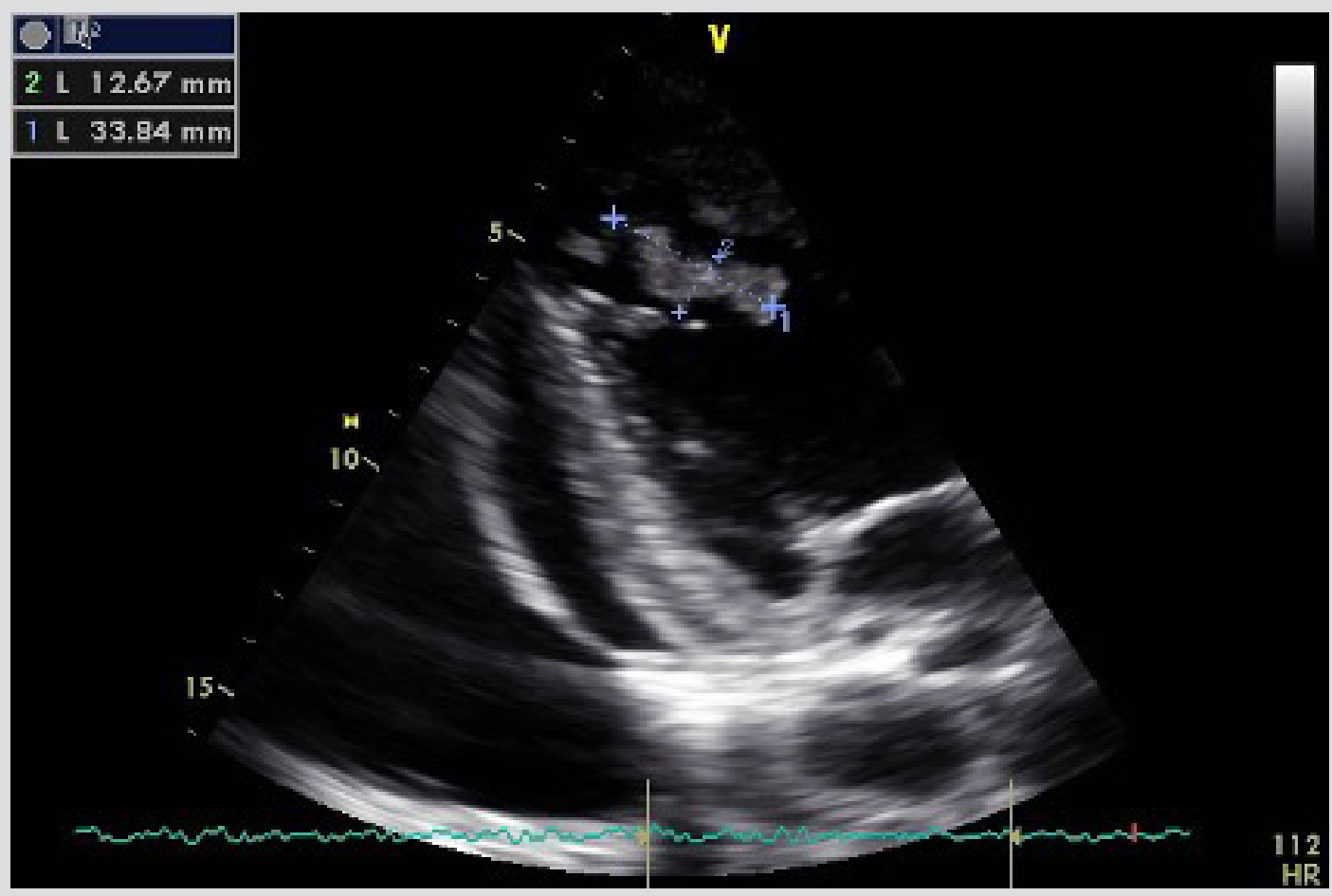

Figure 1. Initial transthoracic two-chamber apical echocardiographic view showing the mass in the left ventricle

Anticoagulation with enoxaparin was initiated and, given the risk of embolization and malignant arrhythmias, the patient was transferred to an intensive care unit where he remained for approximately 48 hours. In this unit, he received a 24-hour infusion of levosimendan. Observation by cardiothoracic surgery personnel excluded indications for surgery, and anticoagulation with enoxaparin was maintained.

Ten days after diagnosis, a new transthoracic echocardiogram was performed and again showed a mass still measuring $30 \times 12 \mathrm{~mm}$, but with overlapping global systolic function. A new ultrasound assessment performed 13 days later showed an undilated left ventricle, with slight hypertrophy and improvement in global systolic function, despite remaining moderately to severely depressed (ejection fraction of $31 \%$ as assessed by Simpson's biplane method) with global hypokinesia. A thrombus with a maximum diameter of $8 \mathrm{~mm}$ was also identified. The thrombus was no longer seen at ultrasound reassessment 2 months later.

The patient was discharged to cardiology care, and is being followed up in an aetiological study of dilated cardiomyopathy. The last transthoracic echocardiogram showed recovery of global systolic function to $39 \%$ and excluded the presence of an intracardiac thrombus. The patient remains anticoagulated with warfarin.

\section{DISCUSSION}

SARS-CoV-2 infection has been associated with a secondary prothrombotic state ${ }^{[1,2]}$ caused by the inflammatory response triggered by the virus, with an estimated risk of thromboembolic events of $25-69 \%{ }^{[3]}$. Left ventricular thrombi are an important complication, mostly associated with the presence of heart failure and ischaemic heart disease, leading to a greater risk of systemic embolic phenomena and death.

In this report, we describe the case of a 50-year-old patient, hospitalized for SARS-CoV-2 infection and congestive heart failure, with the inaugural diagnosis of dilated cardiomyopathy. When transthoracic echocardiography was performed, a thrombus was detected in the left ventricle. The presence of dilated cardiomyopathy with a reduced ejection fraction is itself a risk factor for thrombi. There was no previous diagnosis of intracardiac thrombus, which may thus be related to the combination of these two pathologies. The patient was initially treated with anticoagulation with low molecular weight heparin (enoxaparin) and later with warfarin, showing clinical improvement and complete resolution of the thrombus.

With this case, the authors would like to highlight the importance of performing transthoracic echocardiography in patients with SARSCoV-2 infection. The European Society of Cardiology (ESC) and the American Society of Echocardiography (ASE) do not recommend the routine performance of transthoracic echocardiography in patients with SARS-CoV-2 infection, stating that such examination may influence patient treatment ${ }^{[4]}$, but do not establish any clinical or analytical criteria. 
A prospective study by the ESC suggests that more than half of the transthoracic echocardiograms performed in patients with COVID-19 show abnormalities, including in patients without previous heart disease and particularly when there are elevated levels of natriuretic peptides and high-sensitivity troponin ${ }^{[5]}$. Treatment changes were seen in $16 \%{ }^{[6]}$ to $33 \%{ }^{[5]}$ of patients evaluated by transthoracic echocardiograms, with elevation of D-dimers and troponin being suggested as a predictor of such changes. The prognostic impact of these treatment changes is unclear.

Transthoracic echocardiography is a cheap, non-invasive test, providing valuable information for clinicians and which can be performed without the need to transfer patients, making it extremely useful in times of pandemic. Although there may be a higher risk of infection for technicians due to proximity to the patient, several societies have made recommendations to maintain the safety of healthcare professionals without impacting patient assessment, such as the use of adequate personal protective equipment, the FoCUS (Focus cardiac ultrasound) approach and the use of easily disinfected equipment.

The presented clinical case shows how electrocardiography was essential for the diagnosis and treatment of this patient, even though it should have been performed earlier, as suggested by the increase in NT-proBNP and troponin.

Thus, the authors consider that transthoracic echocardiography should be strongly considered in patients with SARS-CoV-2 infection, especially in the presence of previous risk factors or suggestions of cardiovascular injury. Further studies should be carried out to determine which patients can benefit most from this approach and to understand the therapeutic and prognostic impact of the echocardiographic findings.

\section{REFERENCES}

1. Kaki A, Singh H, Cohen G, Schreiber T. A case report of a large intracardiac thrombus in a COVID-19 patient managed with percutaneous thrombectomy and right ventricular mechanical circulatory support. Eur Hear J Case Rep 2020;4(6):1-5. doi:10.1093/ehjcr/ytaa308

2. Wang T, Chen R, Liu C, Liang W, Guan W, Tang R, et al. Attention should be paid to venous thromboembolism prophylaxis in the management of COVID-19. Lancet Haematol 2020;7(5):e362-e363. doi:10.1016/S2352-3026(20)30109-5

3. Reid N, Carey D, Lang M, Som A, Cochran RL, Alkasab T, et al. Intracardiac and aortic thrombi in the setting of SARS-CoV-2 infection. Eur Hear J Case Rep 2020;4(FI1):1-2. doi:10.1093/ehjcr/ytaa199

4. Skulstad H, Cosyns B, Popescu BA, Galderisi M, Di Salvo G, Donal E, et al. COVID-19 pandemic and cardiac imaging: EACVI recommendations on precautions, indications, prioritization, and protection for patients and healthcare personnel. Eur Heart J Cardiovasc Imaging 2020;21(6):592-598. doi:10.1093/ehjci/jeaa072

5. Dweck MR, Bularga A, Hahn RT, Bing R, Lee KK, Chapman AR, et al. Global evaluation of echocardiography in patients with COVID-19. Eur Heart J Cardiovasc Imaging 2020;21(9):949-958. doi:10.1093/ehjci/jeaa178

6. Benyounes N, Van Der Vynckt C, Tibi S, Iglesias A, Salomon L, Gout O, et al. Echocardiography in confirmed and highly suspected symptomatic COVID-19 patients and its impact on treatment change. Cardiol Res Pract 2020;2020:4348598. doi:10.1155/2020/4348598. 